\title{
Warum nur MRSA?
}

\author{
Winfried V. Kern
}

In Freiburg interessieren wir uns unter anderem für Staphylococcus aureus-Infektionen. Das tun andere natürlich auch. Auch sie wissen: S. aureus-Infektionen sind häufig. Alleine die im Krankenhaus entdeckten bakteriämischen Infektionen - eine der schwersten Verlaufsformen - treten in einer Größenordnung von 20 bis 30 Fällen pro 100000 Einwohner und Jahr auf (Vergleich Tuberkulose, 10/100000, Lungenarterienembolie 100/100000) - in Deutschland sind dies 20000 Fälle jährlich. Die Sterblichkeit ist hoch. Sie beträgt $15-30 \%$. Es ist nicht einfach, die Sterblichkeit zu reduzieren. Wie andere [1 - 5] bemühen wir [6] uns, über intensivierte infektiologische Konsiliartätigkeit das Management zu optimieren - viele Prognosefaktoren sind jedoch nicht - wie z.B. Alter - oder kaum zu beeinflussen. Einige Patienten kommen zu spät in die Klinik; darunter sind einige, die zu früh entlassen worden sind.

Ich erinnere mich noch gut an den 70-jährigen älteren Herrn mit der großen Familie - sehr freundliche Leute. Der Ehemann, Bruder, Onkel und mehrfache Großvater hatte eine koronare Herzkrankheit, und es war eine Herzkatheteruntersuchung mit Angioplastie und Stenteinlage notwendig geworden. 7 Tage nach seiner Entlassung, 10 Tage nach dem Eingriff, werde ich in die Notaufnahme gerufen. Ob der ehemalige Herz-Patient mit hohem Fieber rasch zu uns auf Station verlegt werden könne, eine Pneumonie und Harnwegsinfektion, ebenso ein erneutes akutes Koronarsyndrom seien ausgeschlossen. Das Fieber sei hoch, der Blutdruck derzeit aber stabil, ein Infektionsfokus nicht sicher auszumachen. Wenn ich die Indikation sähe, könne man auch ein Antibiotikum noch in der Notaufnahme starten, nicht, damit es im Rahmen der Verlegung nicht noch zu einer Verzögerung komme. Einige Stunden später ist das Fieber weg, aber der Patient spricht nicht mehr klar, die Urinausscheidung geht zurück, der Blutdruck lässt sich nur mit erheblichen Mengen parenteraler Flüssigkeit halten. Noch vor Ende des nächsten Tages stirbt der freundliche Herr, nachdem die Angehörigen ihn noch mal sehen konnten, im septischen Schock. Klar: S. aureus war in allen Blutkulturen; klar auch: eine adäquate Antibiotikatherapie war innerhalb $2 \mathrm{~h}$ nach Aufnahme begonnen worden; es war nicht MRSA, es waren oxacillin-empfindliche Staphylokokken.

In Freiburg sehen wir bei erwachsenen Patienten pro Jahr 80 - 100 S. aureus-Bakteriämien. Knapp 50\% davon sind nosokomial. Die restlichen Fälle sind formal nichtnosokomiale Fälle, aber immerhin sind $20 \%$ mit medizinischen Maßnahmen assoziierte, im ambulanten Setting symptomatisch gewordene Infektionen. „Nur“ rund $30 \%$ scheinen echte ambulant erworbene Infektionen zu sein. MRSA haben wir hier nicht viel, $10-15 \%$; in einem Jahr waren es bei den BakteriämieIsolaten auch mal 16\%. Viele der schweren S. aureusInfektionen fallen aus der MRSA-Statistik raus, auch die unseres freundlichen alten Herrn - obwohl er eine schwere Infektionskomplikation bei einer Krankenhausbehandlung hatte - mit, nota bene, selbst für Freiburger Verhältnissen hohem Risiko eines tödlichen Ausgangs. Nun ist er nicht und nirgendwo in der MRSAStatistik, aber mit schlechtem Behandlungsergebnis in unserer S. aureus-Bakteriämiestatistik. Übrigens: auch an anderen Orten ist die S. aureus-Bakteriämie häufig, in rund $50 \%$ nosokomial und in $20-30 \%$ zwar ambulant, jedoch mehr oder weniger direkt assoziiert mit medizinischer Behandlung [7-11].

Apropos Statistik: bakteriämische MRSA-Infektionen sind ja inzwischen meldepflichtig. Wie viele solcher schwerer S. aureus-Infektionen sind denn nun gemeldet? Auf der Homepage des RKI finde ich keine Angaben, im „Ländle“ - gemäß Homepage des baden-württembergischen Landesgesundheitsamtes - zählt man diese Infektionen unter der Rubrik „WBK“, weitere bedrohliche Krankheiten, zusammen mit C. difficileInfektionen. Ich finde für die letzten 3 Monate (November, Dezember, Januar) 25 „WBKs“ ohne weitere Differenzierung (außer nach Landkreis), weniger Fälle als Tuberkulose-Neuerkrankungen. Die ganze Aufregung dafür? Alles gut also? Vielleicht 10-12 MRSABakteriämien pro Quartal im ganzen Ländle? Warum (nur) MRSA? Interessiert sich denn tatsächlich niemand für unseren freundlichen alten Herrn und die vielen anderen Infektionen durch oxacillin-empfind- 
liche Staphylokokken als medizinische Komplikation mit Präventionspotenzial?

Ich muss an den Artikel von Dendle und Kollegen [12] denken. Die Kollegen haben 131 Fälle von „health careassociated“ S. aureus-Bakteriämie identifiziert, davon waren $69 \%$ oxacillin-empfindliche Staphylokokken, $73 \%$ waren stationäre Fälle, $50 \%$ assoziiert mit Gefäßkathetern - macht 1,1 Episoden pro 1000 Entlassungen. Die Kollegen waren in der Lage, innerhalb von eineinhalb Jahren diese Rate auf 0,5 Episoden pro 1000 Entlassungen zu reduzieren. Sie betonen, dass für den Erfolg des Projektes der Fokus auf (alle) S. aureus-Bakteriämien im Sinne einer präventiv angehbaren Komplikation medizinischer Behandlung gelegt werden musste - nicht nur MRSA! Die Kollegen sind aus Australien. Dort gibt es übrigens eine Art SAB-KISS - eine webbasierte Erfassung von bakteriämischen S. aureusInfektionen (nicht nur MRSA!). Wie von dort berichtet wurde [13], ist bei einer ersten (multivariaten) Analyse von 1994 (!) solcher Infektionen gefunden worden, dass Alter, schwere Sepsis, Lungen-/Thoraxbeteiligung, Fremdkörperinfektion mit metastatischer Absiedlung, Linksherzendokarditis und Glykopeptidbehandlung, nicht jedoch MRSA vs., MSSA Risikofaktoren für einen tödlichen Ausgang der S. aureus-Bakteriämie waren.

Die australischen Kollegen haben schon vor einigen Jahren gesagt: die S. aureus-Bakteriämie könnte sich gut als Qualitätsindikator für den Krankenhausbereich eignen [14]. Jetzt sagen sie: die Zeit dafür ist gekommen [15].

\section{Literatur}

1 Fowler Jr VG, Sanders LL, Sexton DJ et al. Outcome of Staphylococcus aureus bacteremia according to compliance with recommendations of infectious diseases specialists: experience with 244 patients. Clin Infect Dis 1998; 27: 478-486

2 Ringberg H, Thorén A, Lilja B. Metastatic complications of Staphylococcus aureus septicaemia - to seek is to find. Infection 2000; 28: $132-136$

3 Kaech C, Elzi L, Sendi P et al. Course and outcome of Staphylococcus aureus bacteraemia: a retrospective analysis of 308 episodes in a Swiss tertiary-care centre. Clin Microbiol Infect 2006; 12: 345-352

4 Jenkins TC, Price CS, Sabel AL et al. Impact of routine infectious diseases service consultation on the evaluation, management, and outcomes of Staphylococcus aureus bacteremia. Clin Infect Dis 2008; 46: $1000-1008$
5 Lahey T, Shah R, Gittzus J et al. Infectious diseases consultation lowers mortality from Staphylococcus aureus bacteremia. Medicine (Baltimore) 2009; 88: $263-267$

6 Rieg S, Peyerl-Hoffmann G, de With K et al. Mortality of Staphylococcus aureus bacteremia and infectious diseases specialist consultation - a study of 521 patients in Germany. J Infect 2009; 59: 232 239

7 Allard C, Carignan A, Bergevin M et al. Secular changes in incidence and mortality associated with Staphylococcus aureus bacteraemia in Quebec, Canada, 1991 - 2005. Clin Microbiol Infect 2008; 14: $421-428$

8 Benfield T, Espersen F, Frimodt-Møller $\mathrm{N}$ et al. Increasing incidence but decreasing in-hospital mortality of adult Staphylococcus aureus bacteraemia between 1981 and 2000. Clin Microbiol Infect 2007; 13: $257-263$

9 Morin CA, Hadler JL. Population-based incidence and characteristics of community-onset Staphylococcus aureus infections with bacteremia in 4 metropolitan Connecticut areas, 1998. J Infect Dis 2001; 184: 1029-1034

10 El Atrouni WI, Knoll BM, Lahr BD et al. Temporal trends in the incidence of Staphylococcus aureus bacteremia in Olmsted County, Minnesota, 1998 to 2005: a population-based study. Clin Infect Dis 2009; 49: e130-138

11 Laupland KB, Ross T, Gregson DB. Staphylococcus aureus bloodstream infections: risk factors, outcomes, and the influence of methicillin resistance in Calgary, Canada, 2000 - 2006. J Infect Dis 2008; 198: $336-343$

12 Dendle C, Martin RD, Cameron DR. Staphylococcus aureus bacteraemia as a quality indicator for hospital infection control. Med J Aust 2009; 191: 389 392

13 Turnidge JD, Kotsanas D, Munckhof W. Staphylococcus aureus bacteraemia: a major cause of mortality in Australia and New Zealand. Med J Aust 2009; 191: $368-373$

14 Collignon PJ, Wilkinson IJ, Gilbert GL et al. Health care-associated Staphylococcus aureus bloodstream infections: a clinical quality indicator for all hospitals. Med J Aust 2006; 184: 404-406

15 Collignon PJ, Cruickshank M. Staphylococcus aureus bacteraemias: time to act. Med J Aust 2009; 191: $363-364$ 